\title{
Oerskovia xanthineolytica bacteremia with hypernatremic seizure in an infant with severe pneumonia: an uncommon but life threatening disease treated in a critical care ward in Bangladesh
}

\begin{abstract}
Sharifuzzaman ${ }^{1}$, Dilruba Ahmed², Syeda Umme Habiba Wahid², Tuhin Sadique ${ }^{2}$, Razib Mazumder ${ }^{2}$, Mohammad Anowar Hossain ${ }^{2}$, Nusrat Jahan Chowdhury ${ }^{3}$, Md. Abdullah-Al-Maruf ${ }^{4}$, Mohammod Jobayer Chisti ${ }^{*}$

Abstract:

A 8 months old mildly underweight baby girl was initially admitted at the Short Stay Ward of Dhaka hospital of icddr,b with acute watery diarrhea. On the next day she was transferred to Intensive Care Unit (ICU) of the same hospital for convulsion. After exclusion of potential differentials of convulsion, hypernatremia was added as new problem. She was treated for AWD and hypernatremic convulsion at the ICU following standard hospital protocol. She had also found to have pneumonia (confirmed by radiological evidence) which eventually improved with second line of antibiotics. Finally she found to have Oerskovia xanthineolytica bacteremia which was improved with the ongoing treatment of pneumonia. We report Oerskovia xanthineolytica bacteremia as the $1^{\text {st }}$ case in Bangladesh.
\end{abstract}

Keywords : Children, seizure, Diarrhea, Hypernatremia, Oerskovia xanthineolytica bacteremia, pneumonia.

\section{Introduction :}

Oerskovia spp., gram positive bacilli, grow in the form of branching filament and produce yellow colonies, resembling Nocardia spp. but which unlike Nocardia spp., fragment into motile rod forms ${ }^{1}$. The genus possesses two species. Oerskovia xanthineolytica and Oerskovia turbata which inhabit in the soil and it has been reported that they are rarely responsible for infection in humans ${ }^{1,5,6}$. Nine of the ten previously reported cases of Oerskovia infection occurred in immunocomprised patients and/or were associated with foreign bodies ${ }^{1}$. Potential infections in human include native valve endocarditis ${ }^{2}$, pyonephrosis \& pneumonia $^{1}$, peritonitis $^{3}$, and prosthetic knee joint infection ${ }^{1}$. To our knowledge no cases of Oerskovia infection has been reported in Bangladesh yet. In this report we describe a patient with acute watery diarrhea (AWD) with convulsion and pneumonia possibly due to Oerskovia xanthineolytica with relevant review of the literature describing human infections caused by Oerskovia spp.

1. Sharifuzzaman, Mohammod Jobayer Chisti. Nutrition and Clinical Services Division (NCSD), International Centre for Diarrhoeal Disease Research, Bangladesh (icddr,b), Dhaka, Bangladesh

2. Dilruba Ahmed, Syeda Umme Habiba Wahid, Tuhin Sadique, Razib Mazumder, Mohammad Anowar Hossain. Laboratory Services and sciences Division, icddr,b, Dhaka, Bangladesh

3. Nusrat Jahan Chowdhury. Infectious Diseases Division, icddr,b, Dhaka, Bangladesh

4. Md. Abdullah-Al-Maruf. Moulvibazar Sadar Hospital, Moulvibazar, Sylhet, Bangladesh

Corresponding Author:

Dr. Mohammod Jobayer Chisti

MBBS, MMed, PhD

Scientist, NCSD \& Clinical Lead, ICU

Dhaka Hospital \& Head Clinical Research, Hospitals, icddr,b 68 Shaheed Tajuddin Ahmed Sarani, Mohakhali, Dhaka 1212 Bangladesh, E-mail: chisti@icddrb.org
Case history :

A 8 month old female baby, only issue of her non-consanguineous parents, delivered normally at home, immunized as per to EPI schedule, hailing from a poor family in Dhaka city, was admitted at Short Stay Ward (SSW) of the Dhaka hospital of icddr,b in January 2013 with the complaints of passage of loose stool with a frequency of 7-8 times a day for 6 days prior to admission with no documented fever but cough for 3 days which was dry. She was exclusively breast-fed and her family members never used sanitary latrine. She received some medication and oral rehydration salt (ORS) solution at home for her present illness but could not mention the exact medication. The baby did not have significant contributing past history. The parents were illiterate and had a total of 6 family members who shared the same tin shed. The baby passed urine 1 hour prior to admission. On admission the girl weighed $7.8 \mathrm{~kg}$ and her weight for age $\mathrm{z}$-score was -1.13 of the median of the World Health Organization (WHO) growth standard. However, her birth weight and height could not be retrieved. Here at SSW, the enlisted problems were AWD and cough.

Soon after admission at SSW, she was transferred to Intensive Care Unit (ICU) of same hospital due to development of generalized seizure that was initially treated with consecutive two doses of inj. Lorazepam but the seizure was finally aborted by a loading dose of inj. Phenobarbitone followed by maintenance dose. After the cessation of seizure in the ICU, the baby was found to be drowsy (most probably due to sedation), radial pulse was $170 / \mathrm{m}$ with good volume, BP$130 / 80 \mathrm{~mm}$ of $\mathrm{Hg}$, temperature was 37.8 degree Celsius, respiratory rate was $64 /$ minute (normal respiration rate, as defined by the WHO, is $<50$ per minute for children aged 2 months to 11 months). However, the baby had no lower chest wall in-drawing or cyanosis and breath sound was vesicular 
without any adventitious sound in lungs, but arterial oxygen saturation without oxygen was $80 \%$ in room air. Her jerks were slightly brisk in all four limbs but there were no other signs of meningisms. Examinations of other systems did not reveal any abnormalities.

On the basis of above history and clinical examination the initial problems enlisted in the ICU were, a) AWD, B) convulsion, C) pneumonia. For convulsion we differentially thought -hypoglycemia, dyselectrolytemia, meningitis. However, her random blood sugar was $6.4 \mathrm{mmol} / \mathrm{L}$. After sending relevant laboratory investigations [complete blood count (CBC), blood culture and sensitivity, serum electrolytes with creatinine, chest $\mathrm{X}$-ray, routine microscopic examination (RME) of stool and urine, cerebrospinal fluid (CSF) study] patient was managed with supplemental oxygen of $2 \mathrm{~L} /$ minute (maintain saturation @ 96\%) and inj. ampicillin and gentamicin. Her total WBC was $17,210 / \mathrm{mm}^{3}$ with Neutrophil $44.4 \%$, Band $4 \%$, serum sodium was $161.7 \mathrm{mmol} / \mathrm{L}$, potassium $3.76 \mathrm{mmol} / \mathrm{L}$, total calcium $1.83 \mathrm{mmol} / \mathrm{L}$, total magnesium $0.75 \mathrm{mmol} / \mathrm{L}$, creatinine $44.4 \mathrm{micromol} / \mathrm{L}$. CSF study revealed glucose was $5.43 \mathrm{mmol} / \mathrm{L}$, protein- $0.30 \mathrm{~g} / \mathrm{L}$, gram stain was normal. Chest X-ray showed the features of pneumonia which was patchy, urine R/M/E - normal, stool $\mathrm{R} / \mathrm{M} / \mathrm{E}-$ normal. Thus, the additional problems were hypernatremia and hypocalcemia and treatment of both of the problems were given following hospital management guideline. Both hypernatremia and hypocalcemia were corrected within 24 hours of initiation of management.

Diarrhea was resolved after 4 days of hospital admission with no further episode of seizure and anticonvulsant was gradually titrated after 48 hours of convulsion free period. However, pneumonia was not responding to initial antibiotics (on the basis of persistence fever, increased work of breathing evidence by fast breathing, cough, and lower chest wall in-drawing) for more than 72 hours. By this time we had also received the blood culture report that revealed Oerskovia xanthineolytica that was sensitive to ceftriaxone. Thus, ceftriaxone was added in place of inj. Ampicillin. As the condition was gradually improving on following day she was transferred to LSW and at her stable condition she was discharged with antibiotics and relevant advices after a total of 6 days of hospital stay

Laboratory procedure: About $2.0 \mathrm{ml}$ blood was collected from the patient with adequate precaution and measure and was processed for culture in BacT ALERT 3D machine. The machine positive blood sample was then streaked onto blood agar and chocolate agar plate. The plates were then incubated under microaerophilic conditions at $35^{\circ} \mathrm{C}$. Biochemical analysis using API Coryne strip (bioMeriux) identified the organism as an Oerskovia spp following manufacturer's instruction. Antimicrobial susceptibility test of the organism was performed by disk diffusion technique using commercially available antibiotic discs on Muller-Hinton agar plates. The susceptibility result was reported following the guidelines of the Clinical and Laboratory Standards Institute (CLSI 2010).

\section{Results :}

After 72 hours of incubation culture plates showed presence of small glistening, yellow, catalase-positive colonies on blood agar plates. Gram staining of these colonies revealed gram-positive branching diptheroid like rods. The colonies were non acid fast organism . At $72 \mathrm{~h}$ the colonies were bright yellow (Figure 1a; 1b) on Trypticase soy agar plate. The organism was microaerophilic, catalase positive, oxidase negative, urease negative and motile. Biochemical test result showed acid production by fermenting glucose, lactose, sucrose, maltose, and xylose. The organism could not ferment mannitol however reduced nitrate to nitrite. Citrate was not utilized. $\beta$-Galactosidase was produced. Gelatin, esculin, casein, xanthine, and hypoxanthine were hydrolyzed.

The antimicrobial susceptibility testing showed that the isolate was resistant to Ceftazidime, Nitrofurantoin, Aztreonam, Clindamicin, Norfloxacin Oxacillin; intermediate resistance to Erythromicin, Tobramicin. The strain was susceptible to ampicillin, amoxicillin-clavulanate, tetracycline, cefotaxime, piperacillin-tazobactam, amikacin, gentamicin, imipenem, chloramphenicol, vancomycin, ceftriaxone, penicillin, doxycycline, cotrimoxazole, Ciprofloxacin and cephalothin. Standard MIC interpretative results were as follows: susceptible to amoxicillin-clavulanate $(\leq 0.75 \mu \mathrm{g} / \mathrm{ml})$, tetracycline $(\leq 0.75$ $\mu \mathrm{g} / \mathrm{ml})$, imepenem $(\leq 1.0 \mu \mathrm{g} / \mathrm{ml})$, vancomycin $(\leq 0.13 \mu \mathrm{g} / \mathrm{ml})$, ciprofloxacin $(\leq 0.75 \mu \mathrm{g} / \mathrm{ml})$, cephalothin $(\leq 1.5 \mu \mathrm{g} / \mathrm{ml})$, and intermediate to erythromycin $(\leq 1.5 \mu \mathrm{g} / \mathrm{ml})$.

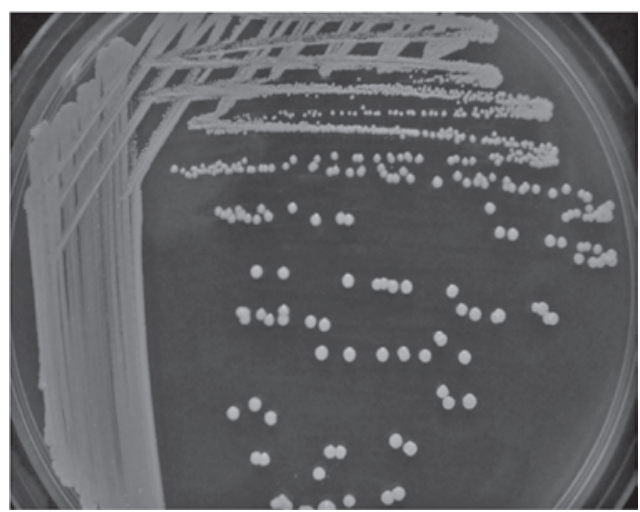

Figure 1a: Bright Yellow colony on Trypticase soy agar plate at $72 \mathrm{~h}$

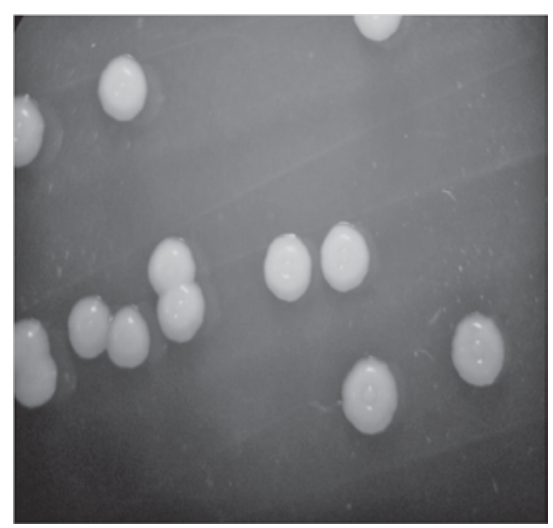

Figure 1b: O. xanthineolytica colonies on Trypticase soy agar plate at $72 \mathrm{~h}$. Magnification, x60. 


\section{Growth and sensitivity of Oerskovia xanthineolytica :}

We observed two important events in our case study. Number one is that Oerskovia xanthineolytica bacteremia with pneumonia is the most important observation in this case and the $2^{\text {nd }}$ was hypernatremia with seizure and both were managed successfully.

Oerskovia spp. isolated occasionally from specimens of laboratory ${ }^{4,7}$. This yellow-pigmented branching organism isolated first by Orskov from the soil in $1938^{4}$. In 1972, O. xanthineolytica was identified by the ability to degrade xanthine and hypoxanthine, in certain reactions of carbohydrate, in the production of phosphatatse ${ }^{1,8}$. In our case, although we do not know whether Oerskovia xanthineolytica was responsible for pneumonia, due to Oerskovia xanthineolytica bacteremia in a case having pneumonia one may speculate that Oerskovia xanthineolytica may be responsible for pneumonia. It is interesting that the pathogen was sensitive to most of the conventional antibiotics. Our patient was eventually receiving inj. ceftriaxone that was sensitive to this bacterium. Although, the course of the disease was eventful, ultimately the patient survived that is consistent to previous all cases with Oerskovia xanthineolytica infection.

Unlikely other reported cases with Oerskovia xanthineolytica infection, our case had diarrhea and hypernatremia. Hypernatremia in diarrheal children usually occurs either due to intake of concentrated or excessive ORS or rotavirus diarrhea! In this case, we do not know the actual reason. However, January is the winter season in Bangladesh (date of admission of our patent was $25^{\text {th }}$ January), rotavirus diarrhea is highly prevalent in that season and it is as high as $70 \%$ of all diarrheal pathogen. Although, we did not look for the rotavirus in our child, normal stool microscopy with plenty of neutral fat suggest the high probability of rotavirus diarrhea in our case. In rotavirus diarrhea, sodium loss in stool is very minimum (30-50 mmol/L), whereas ORS contains $75 \mathrm{mmol} / \mathrm{L}$ sodium. Thus, even with appropriately prepared ORS a patient may develop hypernatremia that potentially occurred in our case. Moreover, fast breathing in pneumonia and fever led to loss of free fluid from the body due to perspiration that might have contributed hypernatremia consequently.

The seizure that occurred in our case might be due to hypernatremia as other potentials causes of convulsion were eliminated. Thus, Oerskovia xanthineolytica infection did not have any direct impact in developing seizure, although remote impact of fever in developing seizure due to Oerskovia xanthineolytica infection could not be ruled out. However, the patient recovered without any documented ramification of hypernatremic convulsion and Oerskovia xanthineolytica bacteremia.In conclusion, the data of our case report suggest that although Oerskovia xanthineolytica infection is uncommon, it may cause bacteremia in diarrheal infant with non-fatal pneumonia, hypernatremia and seizure. However, we need further research to gather more information on Oerskovia xanthineolytica infection especially in diarrheal children.

\section{References :}

1) Harrington $\mathrm{RD}^{1}$, Lewis CG, Aslanzadeh J, Stelmach P, Woolfrey AE. Oerskovia xanthineolytica infection of a prosthetic joint: case report and review. J Clin Microbiol. 1996 Jul;34:1821-4.

2) Ellerbroek $P^{1}$, Kuipers $S$, Rozenberg-Arska M, Verdonck LF, Petersen EJ. Oerskovia xanthineolytica: a new pathogen in bone marrow transplantation. Bone Marrow Transplant. 1998 Sep;22:503-5.

3) Rihs JD ${ }^{1}$, McNeil MM, Brown JM, Yu VL. Oerskovia xanthineolytica implicated in peritonitis associated with peritoneal dialysis: case report and review of Oerskovia infections in humans. J Clin Microbiol. 1990 Sep;28:1934-7.

4) Maguire $\mathrm{JD}^{1}$, McCarthy $\mathrm{MC}$, Decker CF. Oerskovia xanthineolytica bacteremia in an immunocompromised host: case report and review. Clin Infect Dis. 1996 Mar;22:554-6.

5) Cruickshank JG, Gawler AH, Shaldon C. Oerskovia species: rare opportunistic pathogens.

J Med Microbiol. 1979 Nov;12:513-515

6) Guss WJ, Ament ME. Oerskovia infection caused by contaminated home parenteral nutrition solution. Arch Intern Med. 1989 Jun;149:1457-1458

7) Sottneck FO, Brown 1M, Weaver RE, Carroll GF. Recognition of oerskovia species in the clinical laboratory: characterization of 35 isolates. Int J Syst Bacteriol 1977;27:263-70

8) Lechevalier MP. Description of a new species, Oerskovia xanthineolytica, and emendation of Oerskovia Prauser et al. Int J Syst Bacteriol 1972; 22:260-4. 